\title{
Structural Modal Analysis for Detecting Open Solder Bumps on Flip Chips
}

\author{
Dathan S. Erdahl ${ }^{1}$, Matthew S. Allen ${ }^{2}$, Charles Ume ${ }^{3}$ and Jerry H. Ginsberg ${ }^{3}$ \\ ${ }^{1}$ University of Dayton Research Institute - dathan.erdahl@udri.udayton.edu \\ ${ }^{2}$ Dept. of Engineering Physics, University of Wisconsin-Madison - msallen@engr.wisc.edu \\ ${ }^{3}$ Georgia Institute of Technology
}

\section{ABSTRACT}

Although flip chips have received wide acceptance as an integrated circuit package, significant manufacturing problems exist with the integrity of the connection between the package and the printed circuit board (PCB). Conventional x-ray, ultrasonic and electronic testing systems have been used to assess the integrity of this connection, however, none of these have proven suitable for detecting open solder bumps between the chip and the board. The inability to detect open solder bumps with traditional methods merits the investigation of new, nondestructive methods for detecting defects in a manufacturing environment.

This work assesses the feasibility of monitoring the vibration characteristics of flip chips to detect open solder joints. Test vehicles with open solder joints were created, and a nondestructive laser ultrasonic system was used to measure the free vibration response of the chips attached to the printed circuit board. The algorithm of mode isolation (AMI) was applied to the vibration response data in order to extract the modal parameters of the chip. The statistical differences between the modal parameters of sets of damaged and undamaged chips were assessed, revealing the ability of the method to determine the location and severity of these defects in the presence of experimental scatter and manufacturing variation. The parameters of the first mode of vibration, especially its mode shape, were found to be much more sensitive to damage than those of a higher frequency mode.

\section{INTRODUCTION}

Increasing demand for smaller, more compact, and increasingly complex electronics is driving manufacturers to adopt new technology to remain competitive. Active components have the greatest ability to add new features and capabilities to devices. Therefore, the size and complexity of active components has been reduced through the adoption of new interconnection technology using small bumps of solder to connect the component to the board. Solder-bumped surface mount components, such as flip chips, ball grid arrays (BGAs), and chip scale packages 
(CSPs), are being adopted for widespread commercial use. These packages use small bumps of solder to connect the active silicon chip to a printed wiring board (PWB), providing decreased package size, greater I/O density, and increases in signal propagation speed. However, changing from wire lead to surface mount technology poses new problems for the inspection of interconnects hidden between active components and the PWB [1]. Open and cracked solder bumps can sometimes be found using electrical testing methods; however, process development, cycle time and necessary equipment are all very expensive. Test pads for electrical testing on a circuit board use space that was saved by implementation of a flip chip, diminishing the advantage of using a solder-bumped device. In addition, electrical testing will pass a part that has non-wet pads, which provide only an intermittent electrical connection. Other traditional inspection methods, such as X-ray, acoustic microscopy and optical methods, have difficulty in detecting all the defects in these interconnects because of their location and size, however, these bumps serve as mechanical and electrical connection between the chip and the board, so detection of manufacturing defects is necessary to ensure reliability in the field $[2,3,4]$.

Traditional inspection methods cannot adequately detect defects in solder-bumped devices. For example, scanning acoustic microscopy (SAM) uses propagating ultrasonic waves to detect defects, such as changes in material thickness, reflections from interfaces that indicate poor adhesion and voids $[5,6,7,8]$. However, the size of solder bumps and the condition of the solder bump-to-board interface prevent reliable detection of poor solder bump interconnection, even though it has been tested on BGA, CSP and flip chip packages [9,10]. In addition, the liquid couplant required for this method makes it unsuitable for online inspections. Automated $\mathrm{x}$-ray inspection (AXI) applies transmission of X-ray through the PWB, and changes in the amplitude of transmitted energy indicate changes in the material between the transmitter and receiver, and this method is commonly used in online applications for inspection of electronic packages $[11,12,13,14]$. Because open solder bumps have only a small air gap, which does not attenuate $\mathrm{x}$-rays, the defects are extremely difficult to image with this technique.

Therefore, there is a need in the manufacturing workplace for an inspection system that can identify defects with solder bumps in a safe, low-cost, fast, and accurate manner. A new inspection system was previously designed and implemented for flip chip devices $[15,16,17]$. The system is based on the principle of laser ultrasound and interferometric techniques. A pulsed laser excites the chip into vibration, and the vibration of the chip's surface is recorded at 
discrete locations using a laser interferometer. Changes in the vibration response from one chip to another indicate changes in the condition of the chip, as long as the excitation and measurement conditions are identical. Although the system has been previously used for flip chips, chip scale packages [16], and multi-layer ceramic capacitors [18, 19], the testing reported in this paper is on a flip chip with smaller, open solder bumps as defects instead of missing bumps.

Defects in the electronics packages were previously detected using metrics created to compare signals from different specimens of the same type of package. Time and frequency domain methods were used, and pattern recognition algorithms were applied to distinguish small changes in the vibration response [20,21]. Although these methods are somewhat effective, alternative signal analysis methods are sought that can more reliably detect small defects and also provide further insight.

This work explores the possibility of applying modal analysis to the responses measured by the inspection system and tracking changes in modal parameters to identify open solder joints on flip chips. The modal analysis approach is general, and hence could potentially be applied to other electronics packages as well. Modal analysis is attractive as part of a damage detection strategy for a number of reasons. First, modal analysis routines are well developed and may more accurately distinguish structural changes from measurement noise or chip to chip variations than the methods applied previously. Second, modal analysis yields a set of modes that provide insight into the dynamics of the problem. For example, the results presented in the following sections examine two candidate modes, revealing that the lower frequency mode is much more sensitive to open solder joints. This information can be used to select the input and measurement locations that are most likely to accentuate the response features that are sensitive to damage, increasing the sensitivity of the detection system. Finally, a wealth of tools exist that can be applied once a modal model has been identified. For example, the model derived via experimental modal analysis (EMA) can be used to validate or update a finite element model of the structure, or to predict the effect that small changes to the mass and stiffness of the structure would have on the response.

In order to evaluate the effectiveness of the structural modal analysis approach for detecting defects in small structures, single-input multiple output (SIMO) vibration data was recorded using the laser ultrasonic system for a 48-bump flip chip package. The vibration 
response from correctly attached flip chips is compared with the vibration responses from flip chips with open solder bumps. Modal analysis is used to discriminate between the groups of chips, identifying the severity and location of open solder bumps between the chip and the board.

\section{EXPERIMENTAL SETUP:}

The ultrasonic system uses lasers for the excitation and the measurement of vibration responses on the surface of the chips. A $6 \mathrm{~ns}$ pulse, Nd:YAG, infrared laser $(1024 \mathrm{~nm})$ is used to generate the vibration. The laser delivers $2.5 \mathrm{~mJ}$ per pulse, through a single optical fiber, at a rate of $20 \mathrm{~Hz}$ to center of the flip chip's surface, as shown in Figure 1. This pulse level was roughly the maximum that could be applied without damaging the surface of the chip, yet the strain it induces on the solder balls is relatively small. The chip is a peripheral array, $6.3 \mathrm{~mm}$ square, 48-bump daisy-chain device. The bumps are $180 \mu \mathrm{m}$ in diameter and are spaced with a pitch of $457 \mu \mathrm{m}$, with twelve centered on each side of the device. A heterodyne interferometer with a resolution of $0.15 \mathrm{~nm}$ and a bandwidth from $25 \mathrm{kHz}$ to $20 \mathrm{MHz}$ was used to record vibration response data at 48 inspection points on the surface of each chip, however, a $2 \mathrm{MHz}$ low-pass filter was used to reduce high-frequency noise [22]. Each vibration response was sampled at $25 \mathrm{MHz}$ with 12-bit resolution to record highly accurate frequency and amplitude data. The vibration responses were averaged 128 times to reduce white noise, maximizing the repeatability of the system. A linear motion stage was used to position the flip chip relative to the location of the interferometer; the stage utilized an automated computer control for repeatable positioning from one chip to the next.

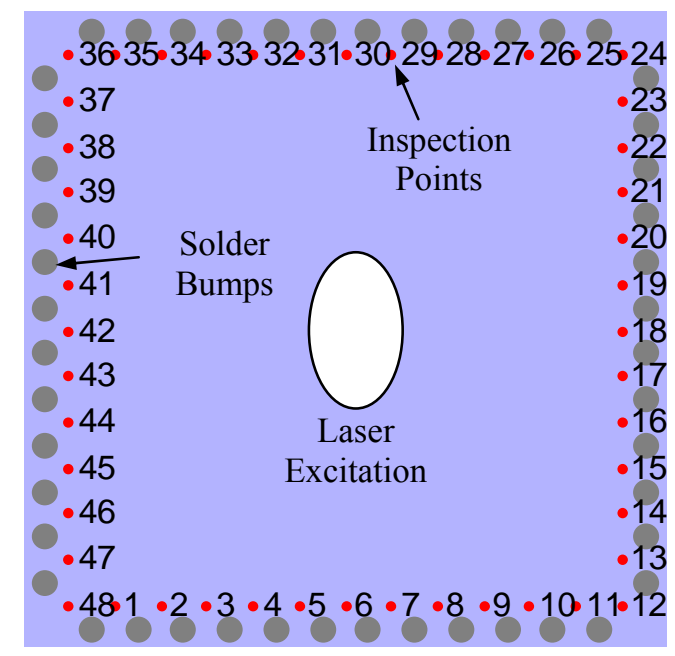

Figure 1: Flip Chip Package with Laser Excitation and Interferometric Measurement Locations 
A total of twenty chips were measured, and all 48 inspection points were sampled on seven separate scans of each chip, creating a vibration response record of each point sampled on the surface. Intentionally created open solder bumps were designed into the test samples in two patterns. The first pattern contained between one and seven open solder bumps in the center of a side. All of these chips were mounted on a single board, along with three reference chips that did not contain any (intentional) defects. A second board was created with chips that had one to seven open solder joints starting at a corner of the chip and progressing towards the center of one side. This board also contained three additional reference chips. The vibration response of chips with intentional defects are compared with the response of chips having no defects. The vibration response data was processed using a Fast Fourier Transform (FFT) algorithm to generate an approximation to the frequency response functions (FRFs) of the chips, which was required as an input to the modal analysis algorithms.

\section{MODAL ANALYSIS}

\subsection{EMA Process Overview}

The modal analysis method utilized in this paper is called the Algorithm of Mode Isolation (AMI). AMI is a procedure by which modes are sequentially identified and subtracted from an experimental data set. Their contributions are then refined through an iterative procedure, which involves isolating each mode in the response and then refitting it to obtain improved estimates for its parameters. AMI was first presented by Drexel and Ginsberg [23] and later extended to a SIMO version in [24-26]. The Single Input Multiple Output (SIMO) version discussed in [26] was used for the data in this study. (AMI was also extended to a Multiple Input Multiple Output (MIMO) version in [27], [28] and [29], and used to process a small subset of the data, yet these results will only be briefly mentioned here.) AMI processes the set of frequency response functions for all input and output measurement points and returns the natural frequency, damping ratio, and mode shape of each vibration mode identified in the data. AMI was chosen because of its ability to process a large number of FRFs quickly, and without introducing spurious or computational modes. The authors also attempted applying the subspace system identification (SSI) algorithm [30, 31], but abandoned it because the time required to process the data with SSI was excessive and it was difficult to identify the modal parameters on the stabilization diagrams that the SSI method generates. 


\subsection{SIMO-AMI Results}

The Algorithm of Mode Isolation was applied to the set of measurements for all 48 outputs. The input power spectrum was assumed to be relatively flat because of the extremely short duration of the excitation force, in which case the Frequency Response Function (FRF) can be approximated by the discrete Fourier transform of the measured responses. Figure 2 shows a composite of the measured frequency response functions (cFRF), a composite of the reconstructed FRF generated using the modal parameters identified by AMI (c(Reconst.)), and a composite of the difference between the two (c(FRF-Reconst.)). The composite FRF is defined here as the average of the magnitude of the FRFs [32]. The reconstructed FRF agrees quite well with the measured FRF over most of the frequency band, indicating that most of the dominant modes in the response have been extracted. Some small peaks are evident in the difference plot, some of which might correspond to weakly excited modes that were not extracted by AMI. A few additional modes could be identified above $900 \mathrm{kHz}$, however, the response above $900 \mathrm{kHz}$ was quite noisy, and so it was decided to focus on the modes below $900 \mathrm{kHz}$.

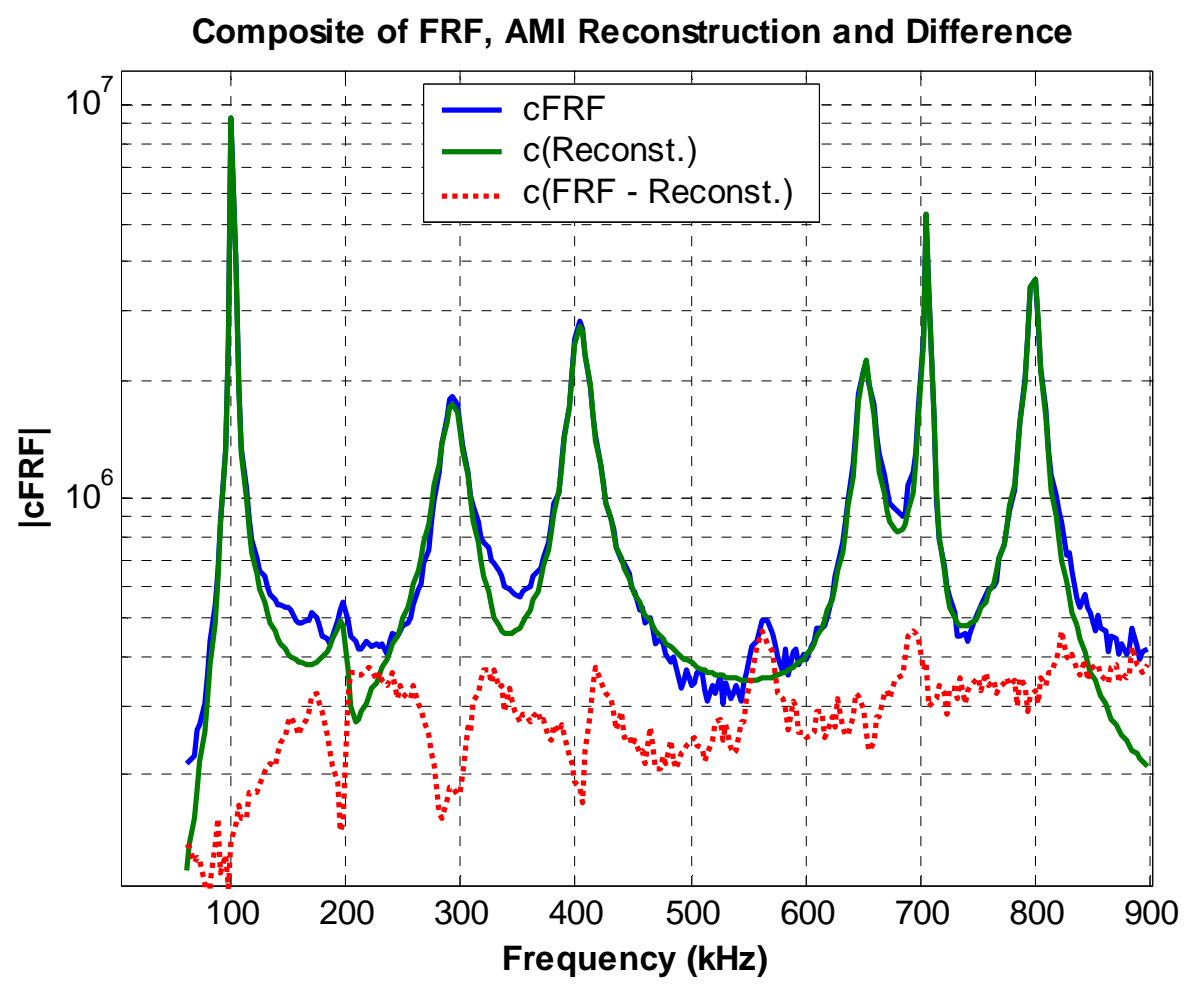

Figure 2: Composite of FRF data and AMI reconstruction for reference chip. 
The natural frequencies and damping ratios found by AMI for the chip-substrate system are shown in Table 1, calculated from data taken on one of the undamaged reference chips.

\begin{tabular}{|c|c|c|}
\hline Mode: & $\begin{array}{c}\mathbf{f n} \\
\mathbf{( k H z )}\end{array}$ & $\boldsymbol{\zeta}$ \\
\hline $\mathbf{1}$ & 101.25 & 0.0090123 \\
\hline $\mathbf{2}$ & 198.85 & 0.023956 \\
\hline $\mathbf{3}$ & 292.93 & 0.03841 \\
\hline $\mathbf{4}$ & 403.58 & 0.020143 \\
\hline $\mathbf{5}$ & 652.19 & 0.010304 \\
\hline $\mathbf{6}$ & 705.79 & 0.0023806 \\
\hline $\mathbf{7}$ & 798.65 & 0.0062361 \\
\hline
\end{tabular}

Table 1: Natural Frequencies and Damping Ratios found by AMI for Reference Chip.

The mode shapes for each of these modes are shown in Figures 3 through 9. The state space description used in AMI accommodates systems in which the damping matrix is not proportional to either the mass or stiffness matrices [33]. Consequently, AMI identifies a complex mode vector for each mode. The damping effects that are dominant on the chips are unknown, as are the characteristics of the input force, and hence the mode vectors cannot be easily scaled to a standard definition. In order to plot the mode shapes, the real displacement response in each mode was found at the instant when it is an overall maximum. This was accomplished by multiplying each mode shape $\{\psi\}$ by $\mathrm{e}^{\mathrm{i} \theta}$, with $\theta$ found to maximize the realness of the product $\{\psi\} \mathrm{e}^{\mathrm{i} \theta}$, and then plotting the real part of $\{\psi\} \mathrm{e}^{\mathrm{i} \theta}$, (solid blue lines in Figures 3 through 9.). The imaginary part of $\{\psi\} \mathrm{e}^{\mathrm{i} \theta}$ is also shown in Figures 3 through 9 by a green dashdot line. This type of representation was developed in [29].

When modes are lightly damped, one typically expects the displacement portion of the state-space mode vectors to be purely real or purely imaginary. In such a mode, every response point moves in phase or exactly 180 degrees out of phase, and the mode is said to have good modal phase collinearity [34]. Inspection of these figures reveals that the imaginary parts of the mode shapes are generally much smaller than the real parts, indicating that most of the modes had good modal phase collinearity. Many of the mode shapes of the chip are visually quite similar to the modes one would expect for a free Euler plate. It is interesting to note that modes 1, 3 and 4 seem to show the plate deforming in a similar pattern (perhaps with some small differences at the corners), the primary difference between the modes being that the net 
displacement of the edge of the plate differs. Mode 2 was weakly excited in the vibration response (Figure 2), making mode shape measurements at $198 \mathrm{kHz}$ more susceptible to noise in the signals. 


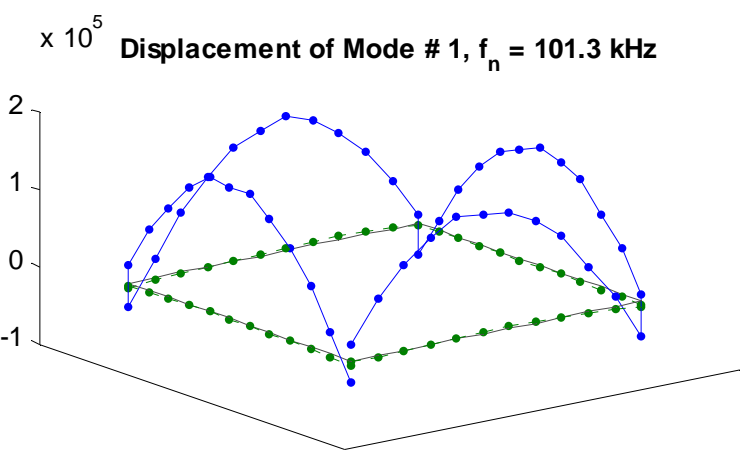

Figure 3: Mode Shape for Mode 1 (Reference Chip)

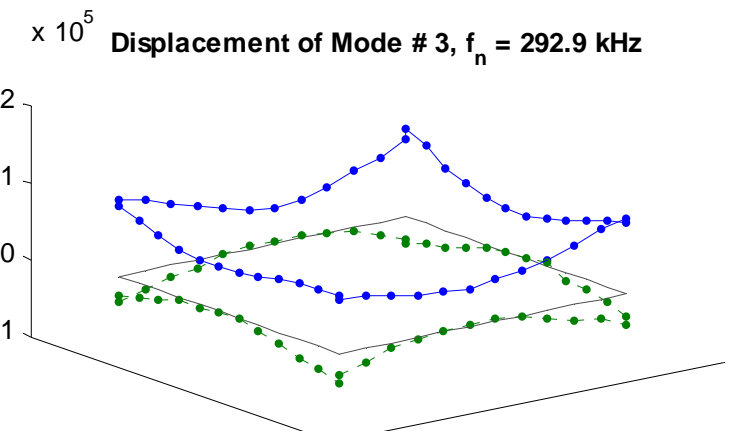

Figure 5: Mode Shape for Mode 3 (Reference Chip)

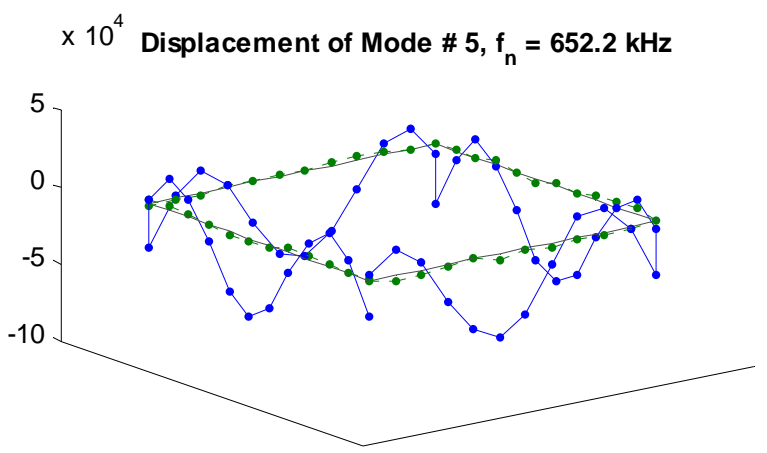

Figure 7: Mode Shape for Mode 5 (Reference Chip)

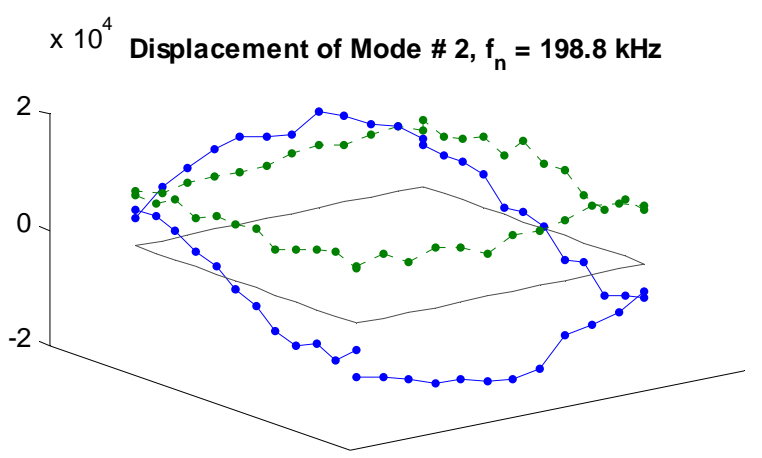

Figure 4: Mode Shape for Mode 2 (Reference Chip)
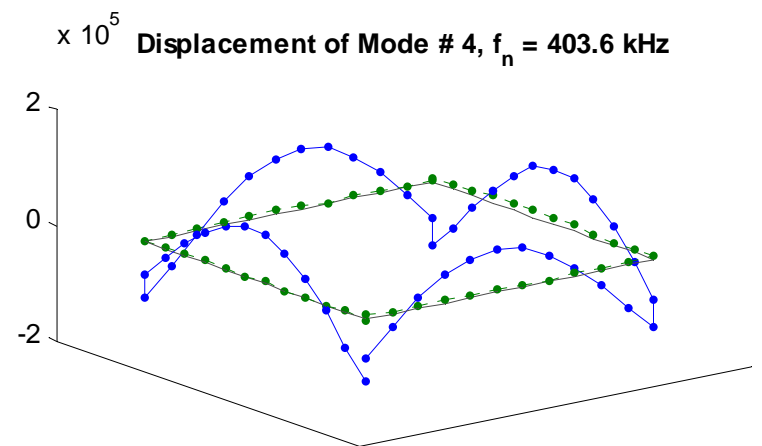

Figure 6: Mode Shape for

Mode 4 (Reference Chip)

$\times 10^{4}$ Displacement of Mode \# 6, $f_{n}=705.8 \mathrm{kHz}$

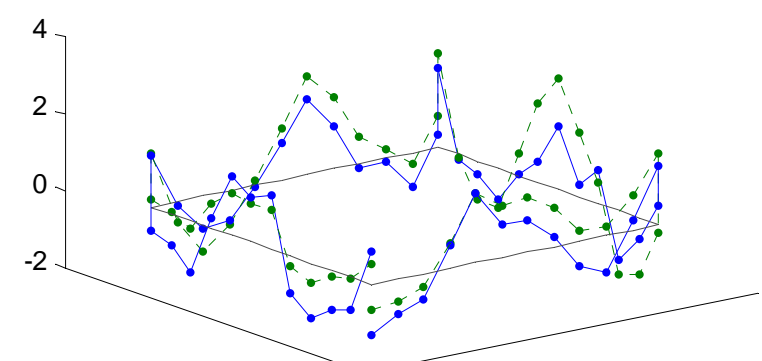

Figure 8: Mode Shape for

Mode 6 (Reference Chip) 


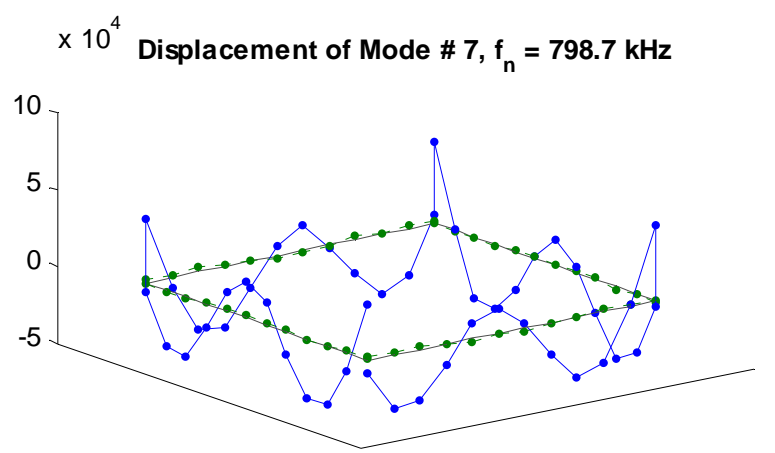

Figure 9: Mode Shape for Mode 7 (Reference Chip)

\subsection{Application of EMA to detect open solder joints}

One common strategy for detecting damage in a structure involves identifying the modal parameters of an undamaged structure and comparing them with the parameters of the structure in question. Differences in the modal parameters of the structure are then correlated with damage to establish detection thresholds. Modes 1 and 7 at approximately 100 and $800 \mathrm{kHz}$ were chosen for this study because they were well excited and easy to extract. Mode 2, near 200 $\mathrm{kHz}$, was weakly excited, preventing usable data from being extracted for all of the experimental sample chips. Mode 2 would be feasible to study in future works if the excitation were moved off center. Modes 3 through 6 were well excited, yet these modes were more difficult to extract from the data. The automatic algorithm described in [29] was able to extract the parameters of modes 1 and 7 without user interaction, greatly simplifying the processing of such a large quantity of data. MIMO testing on a limited number of chips (not reported here) provided an explanation for this difficulty; it indicated that other modes were present in the data with natural frequencies that were close to the natural frequencies of each of modes 3 through 6 . As a result, one could not tell if each of modes 3 through 6 was indeed a distinct mode or a linear combination of two modes with close natural frequencies.

In order to successfully detect the presence of open solder bumps, the modal parameters for the reference chips (no open bumps) must be statistically different from the values for the chips with open solder bumps. The natural frequency values for the $100 \mathrm{kHz}$ and $800 \mathrm{kHz}$ modes are presented in Figure 10. The seven trials for each chip are plotted together, and a normal distribution curve extending two standard deviations from the mean has been fit to the data and is plotted directly above the data values, illustrating the statistical distribution of the values. For the reference chips, the mean and standard deviation were computed from the results 
of testing two different undamaged chips, which were at the same location on two different circuit boards. Any differences between the distributions of these reference chips should indicate a dependence of the measurements on the location of the chip on the board. This dependence was not found to be very significant for these tests, as discussed subsequently, so one can consider the union of the distributions for the reference chips an estimate of experimental scatter for an undamaged chip. To detect damage, one would then create a statistical model of the scatter in the natural frequencies of the reference chips and then compute the probability of getting a particular measurement from a chip under question. Measurements that have low probability (are unlikely) based on the statistical model for the reference set then indicate damage. A detailed statistical analysis was not performed at this stage in this work, but it is relatively easy to assess the situation visually in Figure 10. When comparing the normal distributions for a set of reference chips to one of the chips under question, a larger the separation of the means relative to the standard deviation leads to more confidence that difference is statistically significant and hence that damage is present.

The distributions of the natural frequencies of the $100 \mathrm{kHz}$ mode for the three reference chips are quite similar, suggesting that there is no significant dependence of the natural frequency on chip location for this mode. When comparing this reference set with the other chips, one observes that the reference set overlaps completely with the distributions for one or two open solder joints, so these are undetectable based on the natural frequency information. The distributions for three or more open solder bumps appear to be significantly different from the reference set, although in most cases they overlap somewhat. As a result, the natural frequency obtained in a single test from one of these chips might have a relatively high probability of belonging to the reference (undamaged) set, causing the damage to go undetected. One could improve the probability of detecting these damaged chips based on the natural frequency information by testing each suspected chip multiple times, yet this might be impractical. Some alternative methods of improving sensitivity are discussed in the remainder of this section.

The information for the $800 \mathrm{kHz}$ mode tells a different story. Two of the reference chips have very consistent natural frequencies, yet the other reference chip shows a large amount of scatter. One should note that the maximum frequency shift observed in this mode over all of the chips was less than its modal half power bandwidth. This means that the FRFs for all of the 
chips would be quite similar. Furthermore, one can expect the scatter in the natural frequency estimated by a system identification routine to be some fraction of the modal half power bandwidth, so one should question whether the shift in the natural frequency that was observed for this $800 \mathrm{kHz}$ mode is in fact significant.
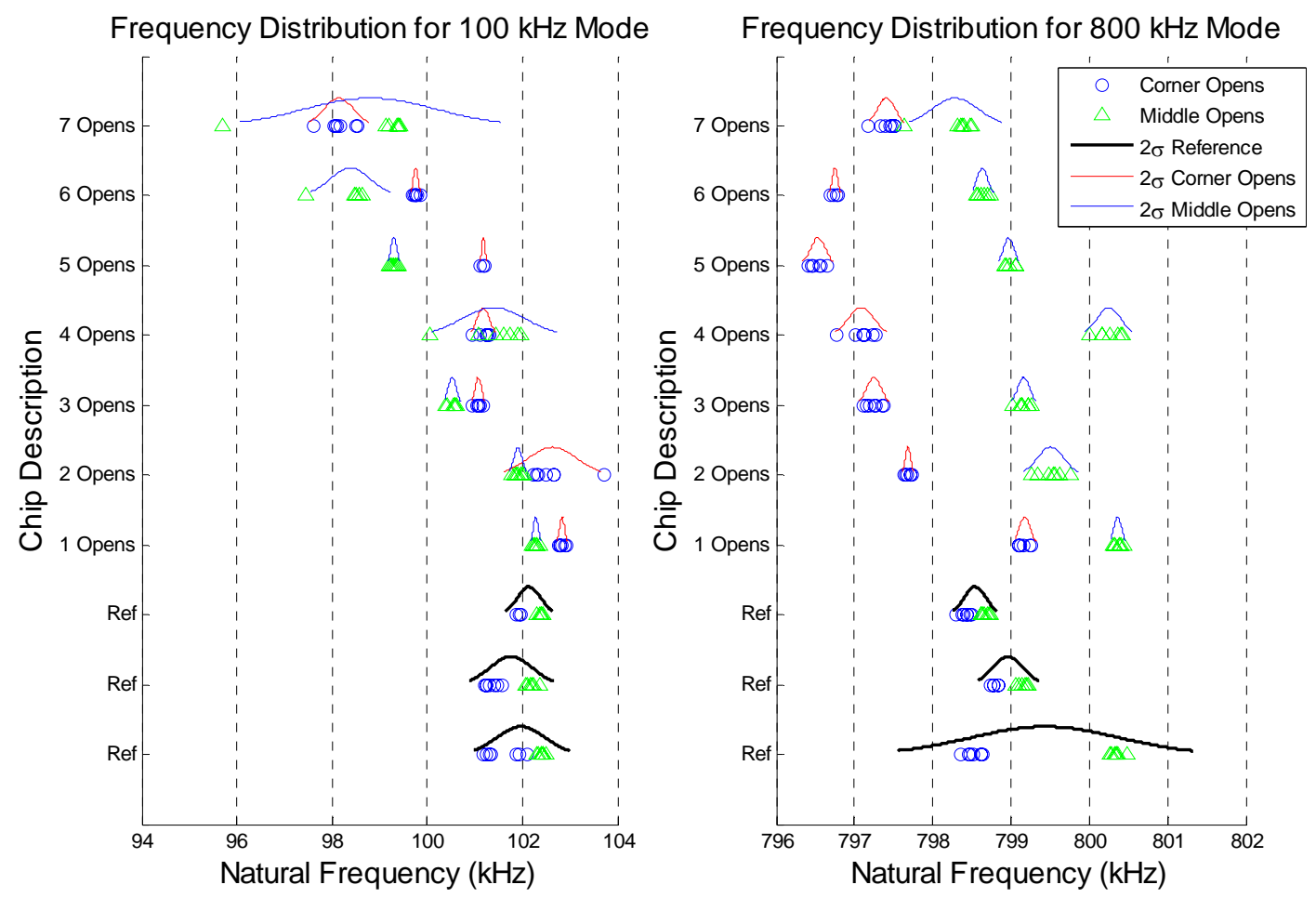

Figure 10: Natural Frequency Distribution for $100 \mathrm{kHz}$ and $800 \mathrm{kHz}$ Modes for 7 Trials on Board Designs 1 and 2.

Using the same format as in Figure 10, the damping ratio values are plotted for all ten chips and the seven trials on each chip for both corner and center opens in Figure 11. The normal distribution is plotted over each set of trials on one chip. The damping ratios for the 100 $\mathrm{kHz}$ and the $800 \mathrm{kHz}$ modes are somewhat less consistent from trial to trial than were the natural frequencies. No coherent distinction is observed between the reference chips and the chips with intentionally open solder bumps. All of the chips have nearly the same overall damping ratio regardless of the number of open solder joints present. 

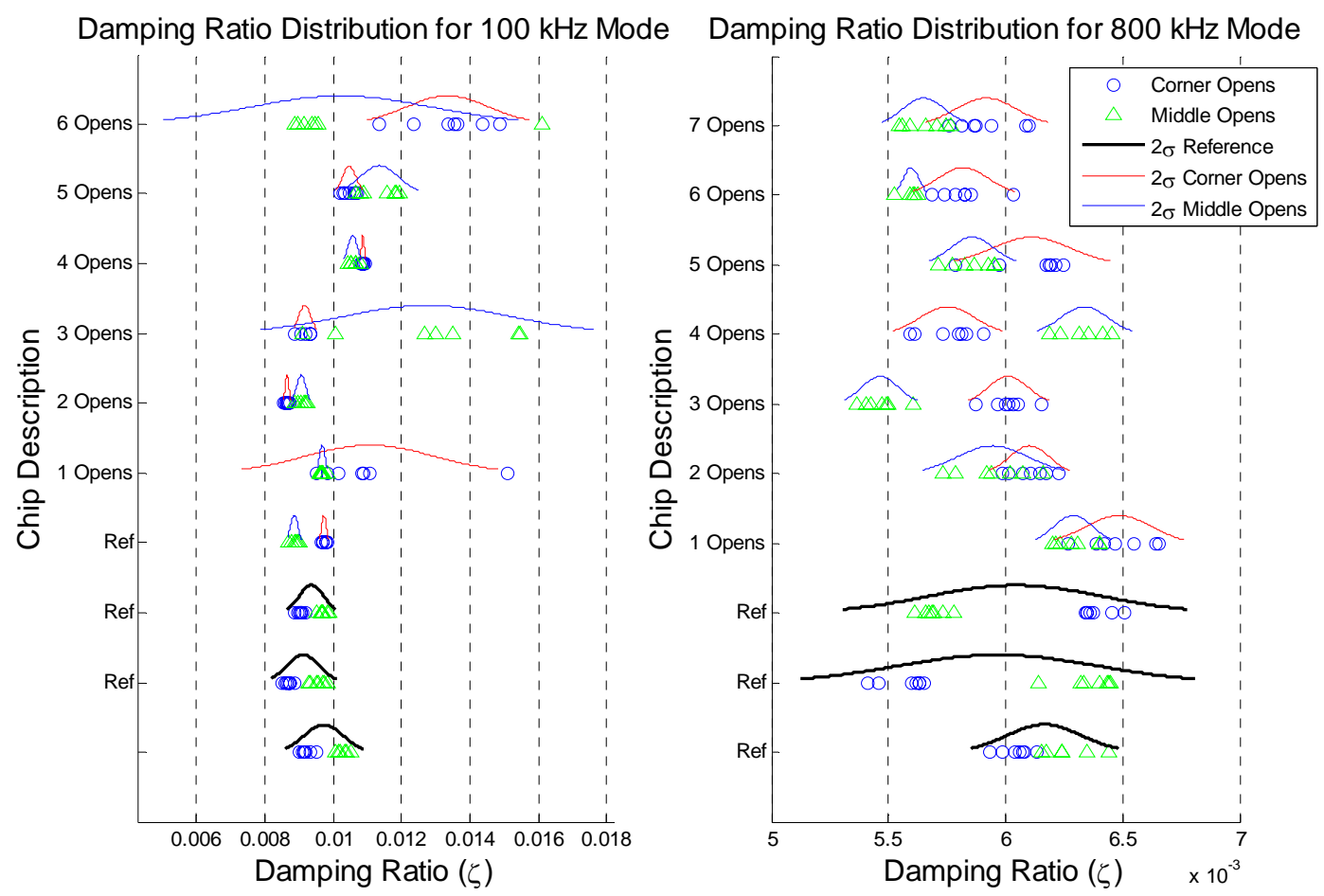

Figure 11: Damping Ratio Distribution for $100 \mathrm{kHz}$ and $800 \mathrm{kHz}$ Modes on Both Board Designs

The modes shapes identified for the chips with and without open solder joints were also compared in order to see if these indicated the presence of damage. Figure 12 shows the average of the $100 \mathrm{kHz}$ mode shape measured in each trial, for each of the chips with open solder bumps in the middle of a side. Figure 13 shows a zoomed in view of the side containing open solder joints. Each defective chip is represented with a colored line, and mode shapes for the three reference chips were averaged together and plotted with positive and negative $2 \sigma$ lines to indicate the level of scatter observed in the mode shape estimate for the reference chips. The highest amplitude and the greatest difference between the average damaged and reference mode shapes occur in the middle of the side, near inspection point 30 . The effect of the open solder bumps is most significant on the side on which the bumps are open, yet there is a visible change in the mode shape on the other sides, especially for those chips with more than three open solder joints. 


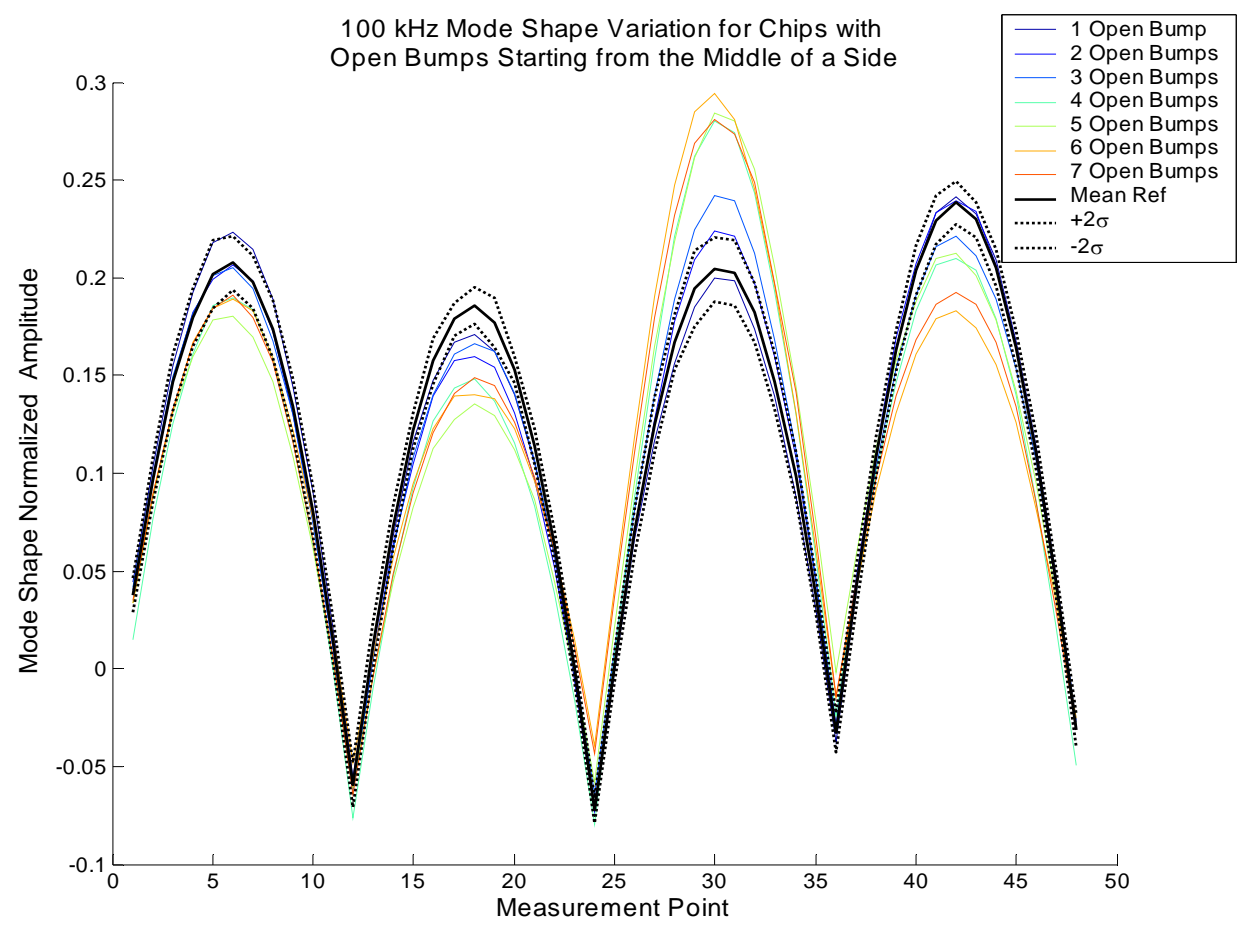

Figure 12: $100 \mathrm{kHz}$ Mode Shape Changes for Chips on Board Design 2 (Open Bumps Start in Middle of the Side Around Measurement Point 30).

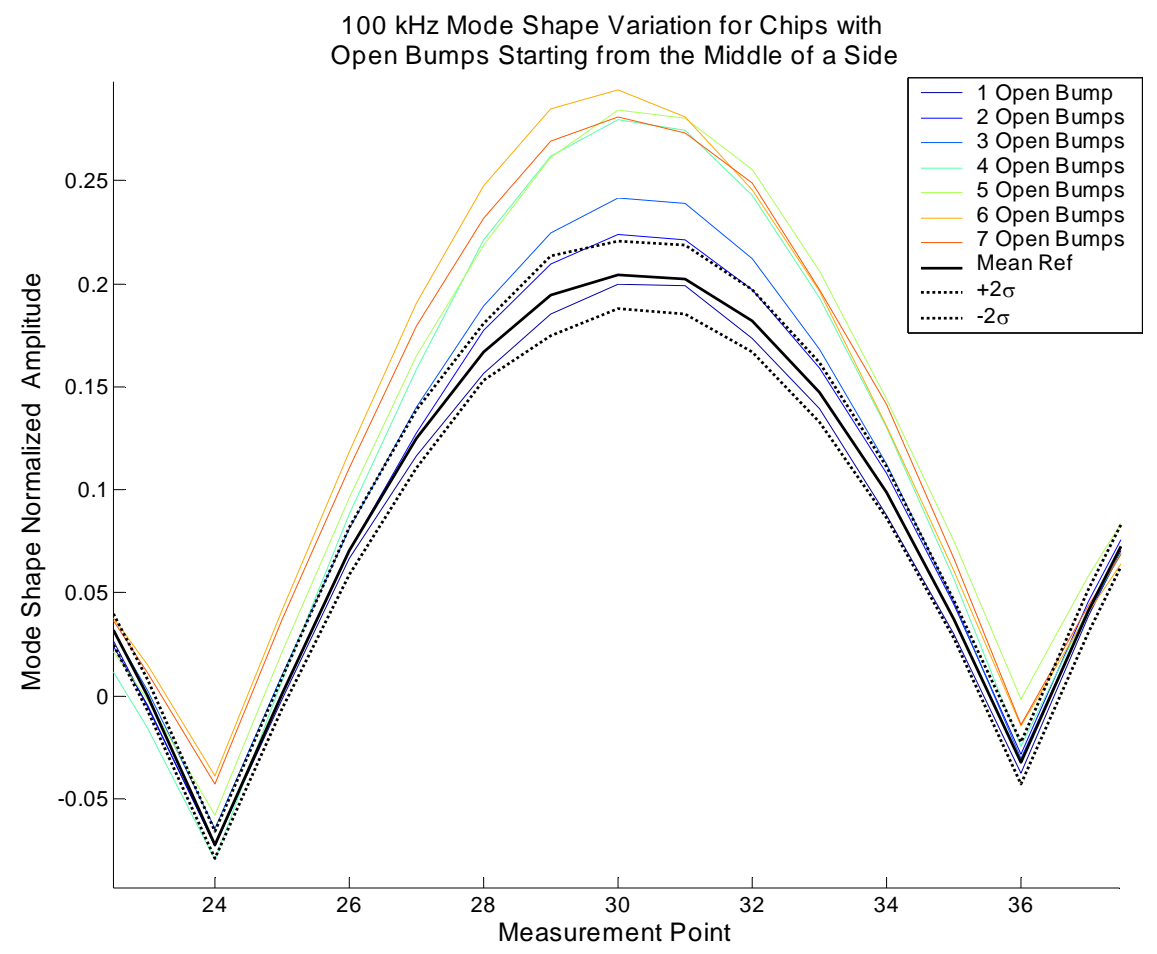

Figure 13: Detail View of Average Reference $100 \mathrm{kHz}$ Mode Shape Compared with All Chips on Board Design 2 Having Intentionally Created Open Bumps in the Middle of the Side Around Measurement Point 30. 
A common technique used to compare mode shapes is the modal assurance criterion or MAC [34]. The MAC is a measure of the linear dependence of two vectors. A value of unity indicates that the two vectors are proportional or parallel, while a value of zero indicates that two vectors are independent or orthogonal (in a Euclidean sense.) For the data shown in Figures 12 and 13 , the $100 \mathrm{kHz}$ mode shapes from all trials on each of the six reference chips were averaged together to form the reference mode shape vector. This reference vector was then compared with the average mode shape for each of the chips, as shown in Figure 14 which shows the MAC values. The reference chips have lower MAC values than most of the chips with open solder bumps. With increasing numbers of adjacent open solder bumps, the changes in mode shape are similar to the changes observed from the mode shape plot in Figures 12 and 13. Three or more adjacent open solder bumps can be identified, but chips with one or two open solder bumps have a MAC value that is similar to the MAC values from the reference chips. Therefore, they may not always be correctly identified. 


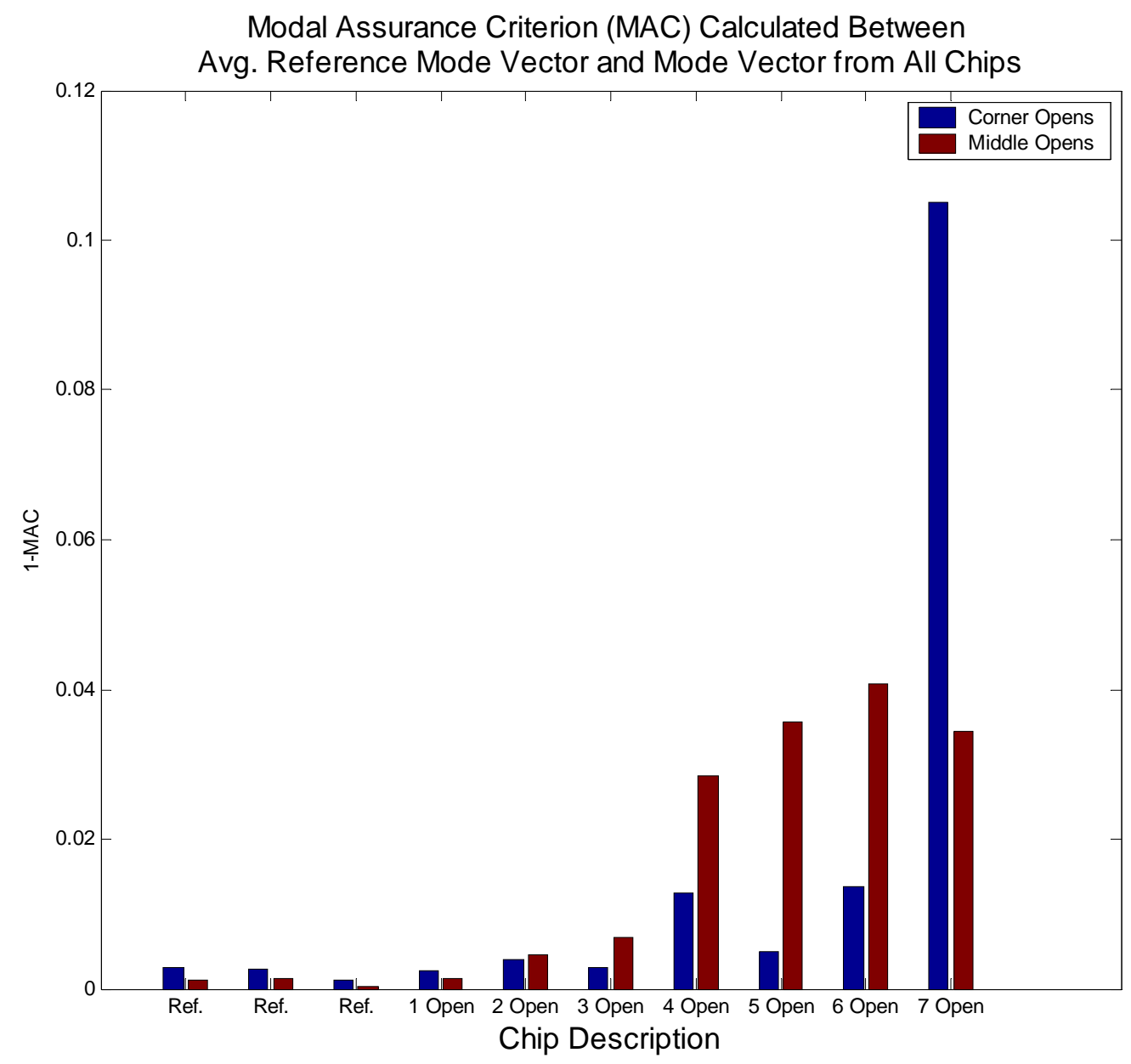

Figure 14: Modal Assurance Criterion (MAC) Values for Every Chip in Comparison to the Average Reference Chip using the $100 \mathrm{kHz}$ Mode.

The mode shapes for the $800 \mathrm{kHz}$ mode are presented in Figure 15a. The detailed view of the region near the open solder bump locations is presented in Figure 15b. The average of all the reference mode shapes is shown with $2 \sigma$ limits, in the same manner as the mode shapes for the $100 \mathrm{kHz}$ mode shown in Figures 12 and 13. All of the mode shapes presented in these plots fall within the $2 \sigma$ limits on the average mode shapes from the reference chips, suggesting that mode 7 was not sensitive to open solder joints. 

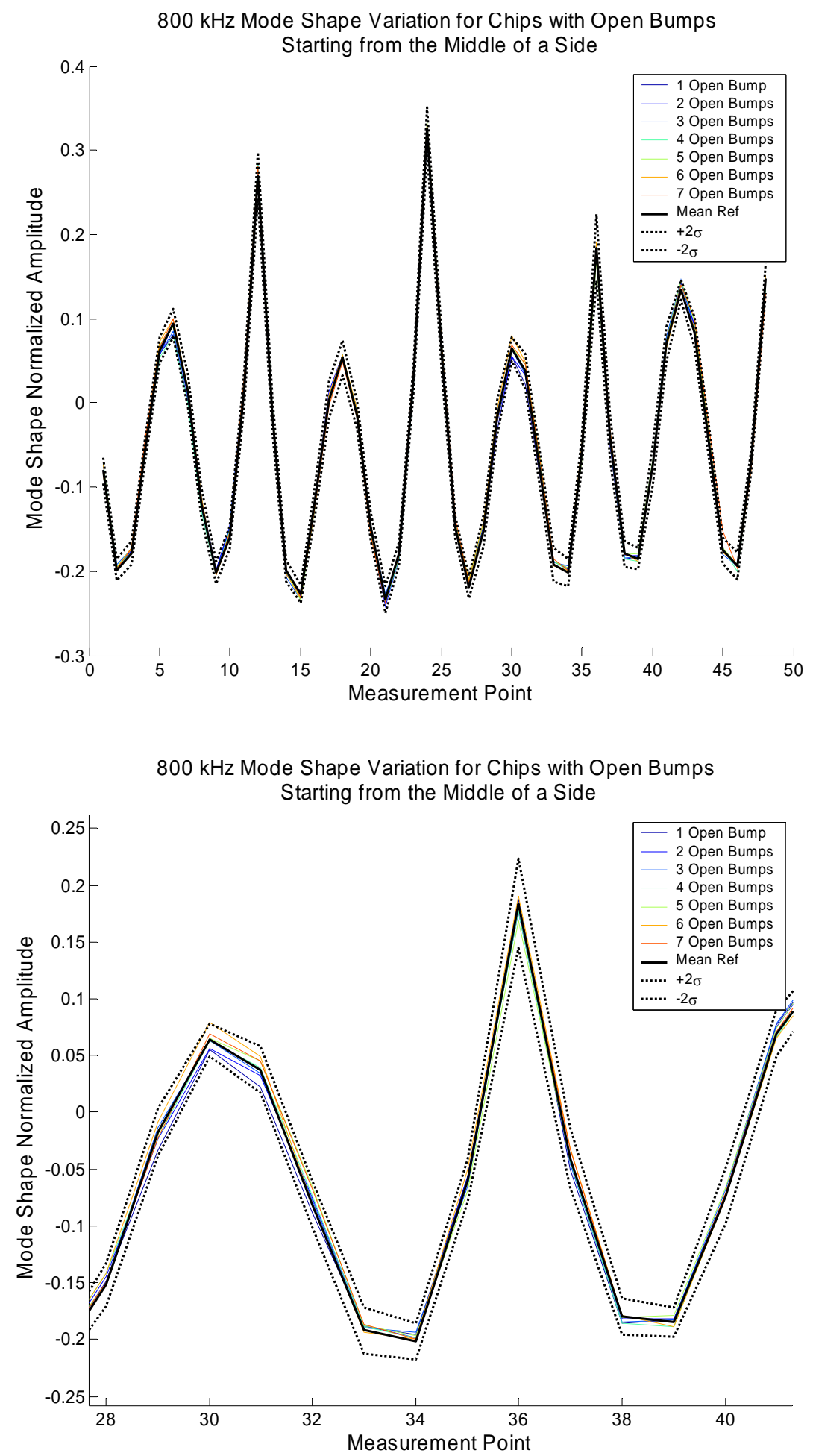

Figure 15: $800 \mathrm{kHz}$ Mode Shape for Chips with Open Bumps Starting from the Middle of the Side Near Measurement Point 30 a.) Full View and b.)Detail View of Location of Open Bumps 


\section{CONCLUSIONS}

The laser-inspection system presented here provides a promising alternative to traditional inspection methods. Implementation of this technique currently requires a significant amount of calibration and refinement, however, the data acquisition and data analysis could be automated relatively easily, resulting in a powerful online inspection system.

The structural modal analysis approach using the AMI method was able to identify the presence of open solder bumps between a flip chip and the circuit board. The most basic approach of monitoring the natural frequency and damping ratio is not definitive in detecting open solder joints because the natural frequencies of the chips with open solder joints are too similar to those for chips without open solder joints relative to experimental scatter. On the other hand, the first mode shape was seen to be significantly different between the reference and damaged chips, so that one could discern many of the damaged chips by comparing the first mode shape of each with the average reference shape. The variation in the shape of a higher frequency mode was also studied, revealing that this mode was not sensitive to damage relative to experimental scatter. The observation that a higher frequency mode was less sensitive to open joints than the $100 \mathrm{kHz}$ mode might indicate that the stiffness of the solder joints becomes negligible relative to the stiffness of the chips at higher frequency. These observations suggest that one should focus the monitoring effort on the low frequency modes. Some preliminary results have been obtained in which the excitation location was varied to better excite the 200 $\mathrm{kHz}$ mode. These results seem to suggest that better detection thresholds might be obtained if both the 100 and $200 \mathrm{kHz}$ modes are monitored. This reveals one important benefit of using modal analysis as part of the damage detection strategy; it sometimes yields insight that can be helpful in designing the detection system.

\section{ACKNOWLEDGEMENTS}

This work was supported by the Engineering Research Program of the Office of Basic Energy Sciences at the Department of Energy, the National Science Foundation, Visteon Corporation, and Siemens. 


\section{REFERENCES}

[1] Oresjo, Stig, 1999. "New Study Reveals Component Defect Levels," Circuits Assembly, May 2002, pp. 39-43.

[2] Sandra L. B. et al, “Automatic Solder Joint Inspection”, IEEE Transactions on Pattern Analysis and Machine Intelligence, Vol. 10, No. 1, 1988.

[3] Chan, Y.C., C.W. Tang, and P.L. Tu, "Endoscopic Inspection of Solder Joint Integrity in Chip Scale Packages," Proc. of the 50th Electronic Components and Technology Conference (ECTC 2000), May 21-24, 2000, Las Vegas, NV, pp. 569-575.

[4] Soden, J.M., R.E. Anderson, and C.L. Henderson, “ IC Failure Analysis: Magic, Mystery, and Science,” IEEE Design \& Test of Computers, July-Sept., 1997, Vol. 14, No. 3, pp. 59-69.

[5] J. E. Semmens, et al. "Further Investigation into the use of Acoustic Micro Imaging for Analyzing Flip Chip Integrity and Failure Modes,” Proc. SPIE, pp.165-169, 1997.

[6] Semmens, J.E., "Flip Chips and Acoustic Micro Imaging: An Overview of Past Applications, Present Status, and Roadmap for the Future," Microelectronics Reliability, Aug.-Oct. 2000, Vol. 40, No. 8-10, pp. 1539-1543.

[7] T. Adams, “Online Inspection for Hidden Internal Defects”, Sensors, pp. 56-61, April, 2000.

[8] L. Bechou, Y. Ousten, and Y. Danto, "New Methods for Scanning Ultrasonic Microscopy Applications for Failure Analysis of Microassembling Technologies," Proc. of the 2001 8th Intl. Symp. on Physical and Failure Analysis of Integrated Circuits, July 9-13, 2001, Singapore, pp. 195-201.

[9] Semmens, J.E. and L.W. Kessler, "Application of Acoustic Frequency Domain Imaging for the Evaluation of Advanced Micro Electronic Packages," Microelectronics Reliability, Sept.-Nov. 2002, Vol. 42, No. 9-11, pp. 1735-1740.

[10] Lawton, W. and J. Barrett, "Characterisation of Chip-on-Board and Flip Chip Packaging Technologies by Acoustic Microscopy," Microelectronics and Reliability, Nov.-Dec. 1996, Vol. 36, No. 11-12, pp. 1803-1806.

[11] Masi, C.G., “The Future of Solder-Joint Inspection”, Test \& Measurement World, July, 1991.

[12] S. Wright, "X-Ray Inspection of IC Packages and PWBs", Chip Scale Review, AugustSeptember, 2001.

[13] Lehman, D.K., "X-Ray Systems for Optimizing PCB Inspection," Circuits Assembly, Feb. 2002, Vol. 13, No. 2, pp. 35-39.

[14] Zweig, G., "Enhance Your BGA X-Ray Inspection Process," Circuits Assembly, May 2002, Vol. 13, No. 5, pp. 47-50.

[15] S. Liu, D. Erdahl, C. Ume, “A Novel Approach for Flip Chip Solder Joint Quality Inspection: Laser Ultrasound and Interferometric System", IEEE Transactions on Components and Packaging Technologies, Vol. 24, No. 4, pp. 616-624, December, 2001.

[16] T. Howard, D. Erdahl, C. Ume, and J. Galmaski, "Development of an Advanced System for Inspection of Flip Chip and Chip Scale Packaging Interconnects using Laser Ultrasound and Interferometric Techniques", The International Journal of Microcircuits \& Electronic Packaging, Vol. 25, No. 1, pp.1-14.

[17] S. Liu and C. Ume, "Vibration Analysis Based Modeling and Defect Recognition for Flip Chip Solder Joint Inspection”, ASME Journal of Electronic Packaging, Vol. 124, Sept., 2002, pp. 221226. 
[18] D. Erdahl, C. Ume, "Online-Offline Laser Ultrasonic Quality Inspection Tool for Multi-Layer Chip Capacitors," Proc. of the 52nd Electronic Components and Technology Conference (ECTC), San Diego, California, May 28-31, 2002.

[19] D. Erdahl, C. Ume, "Laser Ultrasonic Quality Inspection Tool for Multi-Layer Ceramic Capacitors," Proc. of the Capacitor and Resistor Technology Symposium (CARTS 2004), San Antonio, TX, March 29-31, 2004.

[20] S. Liu and C. Ume, "Defects Pattern Recognition for Flip Chip Solder Joint Quality Inspection", Proc. of the 52nd Electronic Components and Technology Conference (ECTC), San Diego, California, May 28-31, 2002.

[21] S. Liu and C. Ume, "Digital Signal Processing in A Novel Flip Chip Solder Joint Defects Inspection System", ASME Journal of Electronic Packaging, Vol. 125, March 2003, pp. 3943.

[22] J. P. Monchalin, "Measurement of In-Plane and Out-of-Plane Ultrasonic Displacements by Optical Heterodyne Interferometry,” Journal of Nondestructive Evaluation, Vol. 8, No. 2, 1989.

[23] M. V. Drexel and J. H. Ginsberg, "Mode Isolation: A New Algorithm for Modal Parameter Identification," Journal of the Acoustical Society of America (JASA), vol. 110, pp. 1371-1378, Sept. 2001.

[24] M. S. Allen and J. H. Ginsberg, "A linear least-squares version of the algorithm of mode isolation for identifying modal properties. Part II: Application and Assessment," Journal of the Acoustical Society of America (JASA), vol. 116, pp. 908-915, 2004.

[25] J. H. Ginsberg and M. S. Allen, "A linear least-squares version of the algorithm of mode isolation for identifying modal properties. Part I: Conceptual development," Journal of the Acoustical Society of America (JASA), vol. 116, pp. 900-907, 2004.

[26] M. S. Allen and J. H. Ginsberg, "A Global, Single-Input-Multi-Output (SIMO) Implementation of The Algorithm of Mode Isolation and Applications to Analytical and Experimental Data," Mechanical Systems and Signal Processing, vol. 20, pp. 1090-1111, 2006.

[27] M. S. Allen and J. H. Ginsberg, "Global, Hybrid, MIMO Implementation of the Algorithm of Mode Isolation," in 23rd International Modal Analysis Conference (IMAC XXIII), Orlando, Florida, 2005.

[28] M. S. Allen and J. H. Ginsberg, "Modal Identification of the Z24 Bridge Using MIMO-AMI," in 23rd International Modal Analysis Conference (IMAC XXIII), Orlando, Florida, 2005.

[29] M. S. Allen, "Global and Multi-Input-Multi-Output (MIMO) Extensions of the Algorithm of Mode Isolation (AMI)," in George W. Woodruff School of Mechanical Engineering Atlanta, Georgia: Georgia Institute of Technology, 2005, p. 129.

[30] P. Van Overschee and B. De Moor, Subspace Identification for Linear Systems: TheoryImplementation-Applications. Boston: Kluwer Academic Publishers, 1996.

[31] P. Van Overschee and B. De Moor, "Continuous-time frequency domain subspace system identification," Signal Processing, vol. 52, pp. 179-194, 1996.

[32] M. S. Allen and J. H. Ginsberg, "A Global, Single-Input-Multi-Output (SIMO) Implementation of The Algorithm of Mode Isolation and Applications to Analytical and Experimental Data," Mechanical Systems and Signal Processing, vol. Under Review, 2005.

[33] J. H. Ginsberg, Mechanical and Structural Vibrations, First ed. New York: John Wiley and Sons, 2001. 
[34] D. J. Ewins, Modal Testing: Theory, Practice and Application. Baldock, England: Research Studies Press, 2000. 